\title{
Colon capsule endoscopy is a viable alternative to colonoscopy for the investigation of intermediate- and low-risk patients with gastrointestinal symptoms: results of a pilot study
}

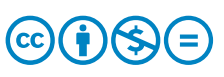

\author{
Authors \\ Deirdre McNamara ${ }^{1,2}$ \\ Institutions \\ 1 Department of Gastroenterology, Tallaght University \\ Hospital, Dublin, Ireland \\ 2 TAGG Research Centre, School of Medicine, Trinity \\ College, Dublin, Ireland \\ 3 Department of Clinical Chemistry, Tallaght University \\ Hospital, Dublin, Ireland
}

Mohd Syafiq Ismail'1,2, Serhiy Semenov ${ }^{1,2}$, Sandeep Sihag1, Thilagaraj Manoharan', Atiyekeogbebe Rita Douglas², Phyllis Reill', Michael Kelly³, Gerard Boran³, Anthony O'Connor ${ }^{1,2}$, Niall Breslin', Sarah O’Donnell', Barbara Ryan',

submitted 26.5.2020

accepted after revision 11.2 .2021

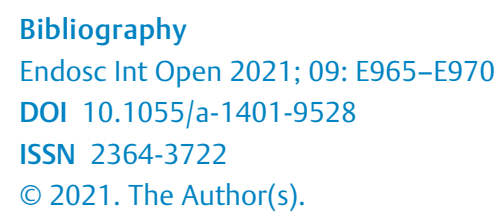

This is an open access article published by Thieme under the terms of the Creative Commons Attribution-NonDerivative-NonCommercial License, permitting copying and reproduction so long as the original work is given appropriate credit. Contents may not be used for commercial purposes, or adapted, remixed, transformed or built upon. (https://creativecommons.org/licenses/by-nc-nd/4.0/)

Georg Thieme Verlag KG, Rüdigerstraße 14,

70469 Stuttgart, Germany

Corresponding author

Mohd Syafiq Ismail, TAGG Research Centre, Trinity Centre,

Tallaght University Hospital, Dublin 24, Ireland

Fax: +35318962988

syafiqismail2009@gmail.com

\section{ABSTRACT}

Background and study aims Colon capsule endoscopy (CCE) is a recommended viable alternative to colonoscopy for colonic visualisation in a variety of clinical settings with proven efficacy in polyp detection, surveillance, screening and Inflammatory Bowel Disease (IBD) assessment. CCE efficacy in an unselected average risk symptomatic cohort has yet to be established. The aim of this study was to determine the feasibility of CCE imaging assessment in average risk symptomatic patients as an alternative to colonoscopy with and without additional biomarker assessment.

Patients and methods This was a prospective, single-center comparison study of colonoscopy, CCE and biomarker assessment.

Results Of 77 invited subjects, 66 underwent both a CCE and colonoscopy. A fecal immunochemical test (FIT) and fecal calprotectin (FC) were available in 56 and 59 subjects. In all $64 \%(n=42)$ had any positive finding with $16(24 \%)$ found to have significant disease (high-risk adenomas, IBD) on colonoscopy. The CCE completion rate was $76 \%$, five $(8 \%)$ had an inadequate preparation, the CCE polyp detection rate was high at $35 \%$. The sensitivity, specificity, positive and negative predictive values of CCE for significant disease were $81 \%$, 98\%, $93 \%$ and $94 \%$ respectively. In addition, three (5\%) significant small bowel diagnoses were made on CCE. FC and FIT were frequently elevated in patients with both colitis $(5 / 7,71 \%)$ and high-risk adenomas (4/7 57\%). While both had a low positive predictive value for clinically significant disease, FIT $32 \%$ and FC $26 \%$.

Conclusions CCE is a safe and effective alternative to colonoscopy in symptomatic average risk patients with or without the addition of biomarker screening.

\section{Introduction}

Colon capsule endoscopy (CCE) is a recommended viable alternative to colonoscopy for colonic visualization in a variety of clinical settings [1]. In keeping with the evidence that patients without alarm symptoms are at low risk of colorectal neoplasia, the European Society of Gastrointestinal Endoscopy guideline of 2012 approved CCE use in average risk patients as a non-invasive option [2,3]. Several non-invasive tests have been evaluated in this setting, including the use of faecal biomarkers, fecal calprotectin (FC) and fecal immunological test (FIT) and other imaging modalities, particularly CT colonography $[4,5]$. 
Biomarkers are now widely incorporated into patient triaging and vetting procedures and are considered a useful means to priorities cases [6,7]. Biomarkers alone can rule-out significant disease in only a minority and imaging tests are still required in the majority of patients to detect both neoplastic and non-neoplastic conditions [4]. As such, evaluation of symptomatic patients still represents a significant burden for colonoscopy services and viable alternatives are needed to help meet increasing demand [8]. There is now an abundance of evidence to show CCE is an effective means to detect both neoplastic and nonneoplastic disease in selected patient cohorts including Inflammatory Bowel Disease patient assessment, average and low risk screening, after incomplete colonoscopy and polyp surveillance [9-15]. However, the efficacy of CCE in an unselected average risk symptomatic cohort, with or without biomarker vetting is less clear. While CT Colonography is now an established alternative in symptomatic assessment, CCE could help to improve overall imaging capacity and offer additional advantages including a simple, non-radiation community-based alternative.

The aim of this study was to determine the feasibility of CCE imaging assessment in average risk symptomatic patients as an alternative to colonoscopy with and without additional biomarker assessment.

\section{Patients and methods}

\section{Population}

This is a prospective, single-center comparative study conducted in Tallaght University Hospital, Ireland. We are the only CCE center in Ireland and perform about 150 to 200 CCEs per year. Following ethical approval, patients aged 18 to 80 years, referred from primary care for investigation of lower gastrointestinal symptoms, who required a non-urgent colonoscopy based on vetting by a Consultant Gastroenterologist applying NICE criteria, were identified [6]. These patients are considered intermediate or low risk. Exclusion criteria for our study were; patients unable to give informed consent or with a contraindication to either study procedure (history of dysphagia, known or suspected small or large bowel strictures, recent abdominal surgery (within 6 weeks)), a contraindication to bowel preparation or allergies to any study medication, patients with coexisting serious medical illness, pregnant subjects, and patients with an ileostomy. Subjects on long-term non-steroidal anti-inflammatories (NSAIDs) could participate if willing to discontinue NSAIDs for 6 weeks prior to study commencement. As per departmental protocol, everyone on regular NSAID's had a patency test to exclude small bowel strictures.

Suitable candidates were invited to participate by phone and a study information leaflet was posted to all potential participants. At the first study visit informed consent was obtained, a medical history, physical examination and routine bloods performed, and study investigations scheduled.

\section{Investigations}

All CCE's were performed using PillCam COLON 2 (Medtronic, Minneapolis, United States). A low-residue diet was advised for 24 hours before the CCE. A standard split bowel preparation re- gimen was used prior to capsule ingestion and booster medications were employed to encourage capsule transit. The study was complete when the capsule was excreted, or the battery ran out. Experienced Capsule Endoscopists, using Rapid Reader Software Version 9, analyzed all videos. Both the first and senior author are approved CCE readers and have performed over 150 and 500 studies respectively. In keeping with our practice, all CCE's were reviewed at our institutions weekly capsule review board. During the period of this study our bowel preparation and booster regimen changed from KleanPrep (Norgine, Middlesex, UK) with Phospho-soda, "Fleet" (Casen Recordati, Zaragoza, Spain.) and Gastrograffin (Bayer, Reading, UK) boosters to MoviPrep (Norgine, Mid Glamorgan, UK ) bowel preparation and boosters. We changed our CCE bowel preparation and booster regimen due to a lack of availability of KleanPrep, having been substituted in our hospital pharmacy with MoviPrep during the study and safety concerns regarding the use of Phospho-soda.

Colonoscopy was scheduled for all patients on average 4 weeks after their CCE. Experienced endoscopists, meeting national quality standards, performed all colonoscopies as a day case. The endoscopist was not blinded to the result of CCE. Colonoscopy reports were generated and stored on a national endoscopy database, Unisoft (HD Clinical, Hertfordshire, UK).

As per our departmental guidelines, for CCE we classified the preparation as being Good, Adequate, or Poor. This is slightly modified from the recommendation from ESGE guidelines for CCE 2012, where we combined the good and excellent rating as Good [3].

For colonoscopy, we had classified prep as being good, adequate/complete, and poor. This is based on pre-assigned prompts from the Unisoft endoscopy reporting system. This system is based on the Aronchick scale.

Significant polyps on CCE were defined, as per ESGE guidelines, as the presence of $>3$ lesions or a polyp greater than $>6 \mathrm{~mm}$ based on polyp size estimation [3]. Significant polyps on colonoscopy was defined as the presence of $>3$ polyps, and adenomas or sessile serrated lesions $>10 \mathrm{~mm}$ in size.

Any histology specimens were processed and reported as normal by our institution's pathology department.

Both FC and FIT samples were collected and delivered within 24 hours directly to the hospital laboratory and processed as standard.

\section{Analysis}

Basic demographics, presenting symptoms, initial blood work, biomarker levels, all CCE and Colonoscopy findings were recorded. For analysis, clinically significant colonic findings included colorectal cancer, high risk adenoma as defined by ESGE and inflammatory bowel disease. Clinically significant small bowel disease was defined as significant ulceration consistent with Crohn's disease or NSAID enteritis, suspicious submucosal masses and $\mathrm{P} 1$ vascular lesions in subjects with a suspicion of bleeding. A FIT of $>10 \mathrm{ug} / \mathrm{g}$ and FC $>50 \mathrm{ug} / \mathrm{g}$ were considered positive.

A per protocol analysis was performed in this study. For comparisons, colonoscopy was considered the gold standard inves- 


\begin{tabular}{|c|c|}
\hline \multicolumn{2}{|c|}{$\begin{array}{c}\text { Recruited } \\
n=77\end{array}$} \\
\hline \multirow[t]{2}{*}{70 Attended for CCE } & Excluded Subjects \\
\hline & $\begin{array}{l}1 \text { withdrew consent } \\
4 \text { did not attend for CCE }\end{array}$ \\
\hline $\begin{array}{c}\text { CCE and Colonoscopy } \\
\begin{array}{c}\text { Performed } \\
N=66\end{array}\end{array}$ & $\begin{array}{l}2 \text { uncontactable and did } \\
\text { not proceed with a } \\
\text { colonoscopy }\end{array}$ \\
\hline $\begin{array}{l}F I T=56 \\
F C=59\end{array}$ & $\begin{array}{l}1 \text { capsule technical } \\
\text { failure }\end{array}$ \\
\hline
\end{tabular}

Fig. 1 Study population.

tigation. CCE findings were compared to colonoscopy and CCE sensitivity, specificity and positive and negative predictive values determined, for any and clinically significant findings. Pearson Coefficient was also used to assess correlation between colonoscopy and CCE findings where an $r$ value of $\geq 0.7$ was considered a strong correlation. Similarly, the accuracy of both FC and FIT was determined.

\section{Ethics approval}

This study was approved by Tallaght University Hospital/St. James's Hospital Joint Research Ethics Committee (REC).

\section{Results}

\section{Study population}

In total, 77 patients were recruited. Of these, one withdrew consent prior to any investigation, two became pregnant after recruitment and four did not attend their CCE appointments. Of the 70 included patients, two did not have a colonoscopy after their CCE as they were not able to be contacted, one patient could not swallow the CCE capsule and in one case there was technical failure of the capsule on ingestion; all of these patients refused a colonoscopy. As a result, there were 66 patients with both CCE and colonoscopy tests available for analysis ( Fig. 1). Only one patient received and passed a patency capsule for a history of chronic NSAIDS use. The mean age of our cohort was 45.8 years (range $20-79)$ and $42 \%(n=27)$ were male. The predominant symptom in 22 (34\%) patients was bleeding, 28 (44\%) diarrhea, seven (11\%) alternating constipation and diarrhea, five (8\%) abdominal pain, two (3\%) weight loss and one (1\%) chronic constipation. As expected only two patients (3\%) had anemia detected on baseline bloods defined as $\mathrm{Hb}<11.2 \mathrm{~g} / \mathrm{dL}$ in women and $<12.8 \mathrm{~g} / \mathrm{dL}$ in men. A C-reactive protein (CRP) was available in 62 patients (97\%) and 56 patients had an erythrocyte sedimentation rate (ESR). In all, 16\% (10/62) had a positive CRP (>5 mg/L). Mean CRP was $4.1 \mathrm{mg} / \mathrm{L}$ (range $1-57.9)$. While, $32 \%(18 / 56)$ had a positive ESR (>20 mm/hr). Mean ESR was 12.5 (1-47) mm/hr.

\section{Colonoscopy performance}

The cecal intubation rate was $94 \%(n=62)$. Bowel preparation was excellent in $39 \%(n=26)$ adequate in $53 \%(n=35)$ and poor in $8 \%(n=5)$. In all, $64 \%(n=42)$ of colonoscopies had a positive finding, with clinically significant disease reported in $24 \%$ $(n=16)$, including two with a histological diagnosis only (one

$\rightarrow$ Table 1 Diagnostic yield by test.

\begin{tabular}{|c|c|c|c|c|}
\hline Parameter N (\%) & Colonoscopy & CCE & (+) FIT & $(+) \mathrm{FC}$ \\
\hline Number & 66 & 66 & $31 / 56$ & $23 / 57$ \\
\hline Completion rate & $94(62)$ & $76(50)$ & $\mathrm{n} / \mathrm{a}$ & $\mathrm{n} / \mathrm{a}$ \\
\hline Inadequate preparation & $5(8 \%)$ & $5(8 \%)$ & $\mathrm{n} / \mathrm{a}$ & $\mathrm{n} / \mathrm{a}$ \\
\hline Normal study & $24(36 \%)$ & $26(39 \%)$ & $\mathrm{n} / \mathrm{a}$ & $\mathrm{n} / \mathrm{a}$ \\
\hline Any positive finding & $42(68 \%)$ & $40(60 \%)$ & $19 / 37(49 \%)$ & $16 / 39(41 \%)$ \\
\hline Clinically significant disease & $16(24 \%)$ & $14(21 \%)$ & $8 / 14(57 \%)$ & $4 / 10(40 \%)$ \\
\hline Polyps & $21(32 \%)$ & $23(35 \%)$ & $\mathrm{n} / \mathrm{a}$ & $\mathrm{n} / \mathrm{a}$ \\
\hline Significant polyp & $7(11 \%)$ & $8(12 \%)$ & $\mathrm{n} / \mathrm{a}$ & $\mathrm{n} / \mathrm{a}$ \\
\hline Colitis & $6(9 \%)$ & $3(5 \%)$ & $\mathrm{n} / \mathrm{a}$ & $\mathrm{n} / \mathrm{a}$ \\
\hline Diverticulosis & $11(17 \%)$ & $14(21 \%)$ & $\mathrm{n} / \mathrm{a}$ & $\mathrm{n} / \mathrm{a}$ \\
\hline Haemorrhoids & $3(5 \%)$ & 0 & $\mathrm{n} / \mathrm{a}$ & $\mathrm{n} / \mathrm{a}$ \\
\hline Microscopic colitis & $2(3 \%)$ & $\mathrm{n} / \mathrm{a}$ & $\mathrm{n} / \mathrm{a}$ & $\mathrm{n} / \mathrm{a}$ \\
\hline Significant small bowel disease & $3(5 \%)$ & $3(5 \%)$ & $\mathrm{n} / \mathrm{a}$ & $\mathrm{n} / \mathrm{a}$ \\
\hline
\end{tabular}


- Table2 Diagnostic accuracy by test compared to colonoscopy for clinically significant disease.

\begin{tabular}{|c|c|c|c|c|c|}
\hline & Test & Sensitivity \% & Specificity\% & PPV\% & NPV\% \\
\hline \multirow[t]{3}{*}{ Clinically significant disease } & CCE & 81 & 98 & 93 & 94 \\
\hline & $\mathrm{FIT}>10 \mathrm{ug} / \mathrm{g}$ & 57 & 60 & 32 & 81 \\
\hline & $\mathrm{FC}>50 \mathrm{ug} / \mathrm{g}$ & 40 & 64 & 26 & 88 \\
\hline \multirow[t]{3}{*}{ Any positive finding } & CCE & 79 & 71 & 83 & 65 \\
\hline & $\mathrm{FIT}>10 \mathrm{ug} / \mathrm{g}$ & 49 & 63 & 72 & 61 \\
\hline & $\mathrm{FC}>50 \mathrm{ug} / \mathrm{g}$ & 41 & 37 & 70 & 66 \\
\hline
\end{tabular}

lymphocytic, one TB colitis). Included as clinically significant disease were six cases of histologically confirmed IBD (3 UC, 3 $(D)$, one case of radiation proctitis, seven patients with highrisk polyps ( 3 adenomas $>10 \mathrm{~mm}$, four $>3$ adenomas and one large $>10 \mathrm{~mm}$ sessile serrated right colon lesion) and both $\mathrm{mi}$ croscopic colitis cases ( $>$ Table $\mathbf{1}$ ).

\section{CCE performance}

In 66 of 68 (97\%) patients referred for CCE videos were available for review and compared to colonoscopy. The excretion rate was $76 \%(n=50)$. Bowel preparation quality was good in $38 \%(n=25)$, adequate in $55 \%(n=36)$ and inadequate in $8 \%$ $(n=5)$. Overall, 40 (61\%) CCE's were positive. There were no procedure related complications ( $>$ Table 1 ). CCE abnormalities included; polyps in 23 (35\%), diverticulosis in 14 (21\%) and Colitis in $n=3(5 \%)$. Overall $n=8(12 \%)$ had significant polyps ( $>3$ lesions or a polyp $>6 \mathrm{~mm}$ ). Any small bowel findings were reported in $n=15$ (22\%) subjects, 3 (5\%) deemed clinically significant and was subsequently diagnosed with Crohn's disease. In all, 14 (21\%) had clinically significant disease detected on CCE. There was one false-positive and three false-negative CCEs in 16 patients with significant disease on colonoscopy. In all, there were two patients with distal colonic inflammation overlooked. In these cases, one had very distal radiation proctitis and the other case the capsule was incomplete. CCE by its nature misclassified the two patients with histologically diagnosed colitis; however, in one of these patients, they also had a significant polyp based on CCE. The only false- positive was accounted for by variation in classification of a polyp based on size. Of note, all significant polyps on colonoscopy reached the CCE threshold for excision. The sensitivity, specificity, positive and negative predictive values of CCE for significant disease were $81 \%, 98 \%, 93 \%$ and $94 \%$ respectively ( $\triangleright$ Table 2 ). The overall correlation between colonoscopy and CCE was moderate, Pearsons $r=0.49$ but was strong for significant disease $r=$ 0.83 .

\section{Biomarker performance}

In all, FIT and FC samples were available for 56 (85\%) and 59 $(89 \%)$ subjects. FIT was positive $(>10 \mathrm{ug} / \mathrm{g})$ in 31 cases $(47 \%)$, range $1-1992 \mathrm{ug} / \mathrm{g}$. Within this cohort there were six false-negative FITs in patients diagnosed with clinically significant dis- ease on colonoscopy $(n=14)$. Overall, the sensitivity, specificity, positive and negative predictive values of FIT for CSD were $57 \%, 60 \%, 32 \%$ and $81 \%$. FC was positive (>50 ug/g) in 23 patients $(40 \%$ ), range $<19.5$ to $1250 \mathrm{ug} / \mathrm{g}$. There were foiur falsenegative FCs in 10 patients with CSD. The sensitivity, specificity, and positive and negative predictive values of FC for CSD were $40 \%, 64 \%, 26 \%$, and $88 \%$, respectively ( Table 2 ). With respect to the seven high-risk polyp cases specifically, four of six $(67 \%)$ returned a positive FIT and four of seven (57\%) a positive FC. Similarly, of the seven cases of colitis on colonoscopy FC was positive in five (71\%), unsurprisingly the FIT was also positive in five patients (71\%) with colitis. Neither CRP nor ESR were predictive of either CCE or Colonoscopy detected disease.

\section{Discussion}

Our pilot prospective single-center analysis of CCE efficacy in an unselected symptomatic cohort suggests CCE is a viable alternative to colonoscopy. As expected only a small proportion of patients were found to have significant disease on colonoscopy, $16(24 \%)$. The majority of patients while needing and often benefiting from a negative test or low-risk diagnosis did not necessarily require an invasive colonoscopy. Viable alternatives, which offer similar diagnostic accuracy and enhanced capacity, would be of benefit to both patients and the health service alike.

After the CCE, colonoscopies were performed on average 4 weeks from the CCE procedure. The rationale for this is that previous blinded studies have assumed CCE findings not detected on the colonoscopy were a false negative, whereas recent evidence suggests that if the negative colonoscopy is repeated, unblinded, many polyps previously overlooked were found on the repeat procedure [11]. In addition, in the real word/clinical scenario, the capsule report is going to be available to the endoscopist before proceeding to colonoscopy.

In our cohort, CCE accurately detected all significant polyps and had a similar diagnostic accuracy for benign disease with PPV's and NPV's of $83 \%$ and $65 \%$ and $93 \%$ and $94 \%$ for any finding and clinically significant disease respectively. However, detection rates for colitis were less than optimal compared to colonoscopy: three of seven (43\%). Only three of seven with colitis on colonoscopy ended up with a diagnosis of UC. This is dif- 
ficult to explain as previous studies and those with modern capsules have found a high rate of diagnostic accuracy in IBD [9, 16]. For very distal limited proctitis the process of excretion may affect video interpretation, with distal inflammation being mis-classified as a normal rectal cushion. Initial analysis of our cohort would support this supposition with two-thirds reclassified as showing disease on repeat reading. Enhanced awareness and training could improve future detection of proctitis and warrants additional investigation.

As expected, CCE was unable to diagnose both cases of microscopic colitis. Neither case had a positive biomarker result. Both had an established history of watery diarrhoea. As such, clinical acumen and the adherence with biopsy protocols in suspected cases with a normal macroscopic colon is important to prevent overlooking microscopic colitis. A negative CCE should not have prevented further referral for biopsy.

Despite the overall good performance of CCE in our study, the capsule excretion rate remains low at $76 \%$. Analysis is in some way hampered by the required change in bowel preparation and booster regimen during this study. Optimising capsule excretion rates remains a priority and like others, we continue to adapt our preparation and booster regimens in line with current data [17]. Following study conclusion, audits of recent changes to our bowel preparation (2 L PEG solution) and booster regimen (PEG booster + Castor oil) has shown an $87 \%$ capsule excretion rate and also improved image quality $[18,19]$. Similarly, improving and standardising reporting of preparation quality in CCE is an area of ongoing research [20]. However, rates of suboptimal/inadequate bowel preparation were similar for both CCE and colonoscopy, five of 66 (8\%), highlighting the importance of this issue for both tests.

As with CT colonography, CCE has the potential to visualise more than just the colon, which may be particularly advantages in patients with chronic gastrointestinal symptoms. In this study, while small bowel abnormalities were detected in 15 ( $22 \%)$ only three $(4.5 \%)$ were significant, all with enteritis, resulting in changed management.

The use of biomarkers is now commonplace in the assessment of patients presenting to hospital or community clinics. While they are helpful indicators of significant neoplasia and inflammatory bowel disease, they are not a replacement for direct colonic visualisation. Our study supports their role in selecting outpatients with a higher risk of clinically significant disease, with five of seven cases of colitis and four of seven with high-risk adenomas having a positive biomarker study. Their low positive predictive value for clinically significant disease, FIT $32 \%$ and FC $26 \%$, suggests referring all positive biomarker patients for a colonoscopy may not be warranted and alternatives such as CCE or CT colonography remain options. For biomarker negative individuals, based on our data, noninvasive imaging may be preferred. Both algorithms warrant further consideration.

The small size of our study is a drawback, but we felt the lack of available evidence for CCE use in a symptomatic unselected cohort warranted a further direct comparison of CCE with colonoscopy. This study design does hamper recruitment. We feel that in the setting of a pilot study assessing a novel aspect of
CCE in investigating the symptomatic patient, where patients had to undergo both CCE and colonoscopy including two sets of bowel preparation, the sample size is adequate to draw some form of a conclusion as a proof of concept. We welcome bigger multi-centred studies in the future following this initial pilot study.

A strength, however, is that endoscopists were unblinded to the CCE result, which may account for the relatively low discrepancy rate in all polyp detection. Previous studies have suggested CCE accurately detects more polyps than colonoscopy, which can later be found on a targeted repeat colonoscopy [11]. As such, it remains a possibility that our colonoscopy polyp detection of 21/66 (32\%) was enhanced in our study by prior CCE. The CCE polyp detection rate was 35\%. Apart from polyp detection, all cases of terminal ileal Crohn's disease were initially picked up on CCE, if an endoscopist does not routinely perform terminal ileal intubation, these cases would have been missed. If borne out in additional studies this would represent another advantage for CCE patient selection.

\section{Conclusion}

CCE is a safe and effective alternative to colonoscopy in symptomatic average risk patients with or without the addition of biomarker screening.

\section{Acknowledgments}

The authors would like to acknowledge the staff in the Capsule Endoscopy Unit and Endoscopy, Tallaght University Hospital.

\section{Competing interests}

The authors declare that they have no conflict of interest.

\section{References}

[1] Spada C, Hassan C, Costamagna G. Colon capsule endoscopy. Gastrointest Endosc Clin N Am 2015; 25: 387-401

[2] Rex DK, Mark D, Clarke B et al. Flexible sigmoidoscopy plus air-contrast barium enema versus colonoscopy for evaluation of symptomatic patients without evidence of bleeding. Gastrointest Endosc 1995; 42: 132-138

[3] Spada C, Hassan C, Galmiche JP et al. Colon capsule endoscopy: European Society of Gastrointestinal Endoscopy (ESGE) Guideline. Endoscopy 2012; 44: 527-536

[4] Graser A, Stieber P, Nagel D et al. Comparison of CT colonography, colonoscopy, sigmoidoscopy and faecal occult blood tests for the detection of advanced adenoma in an average risk population. Gut 2009; 58: 241-248

[5] Mowat C, Digby J, Strachan JA et al. Faecal haemoglobin and faecal calprotectin as indicators of bowel disease in patients presenting to primary care with bowel symptoms. Gut 2016; 65: 1463-1469

[6] National Institute for Health and Care Excellence (NICE). Suspected cancer: recognition and referral. 2018: https://www.nice.org.uk/ guidance/ng12/chapter/1-Recommendations-organised-by-site-ofcancer\#lower-gastrointestinal-tract-cancers 
[7] National Institute for Health and Care Excellence (NICE). Faecal calprotectin diagnostic tests for inflammatory diseases of the bowel. 2013: https://www.nice.org.uk/guidance/dg11

[8] Ismail MS, Aoko O, Sihag S et al. Lower gastrointestinal symptoms and symptoms-based triaging systems are poor predictors of clinical significant disease on colonoscopy. BMJ Open Gastroenterol 2020; 7: e000221

[9] Hosoe N, Hayashi Y, Ogata H. Colon capsule endoscopy for inflammatory bowel disease. Clin Endosc 2020: doi:10.5946/ce.2019.156

[10] Holleran G, Leen R, O'Morain C et al. Colon capsule endoscopy as possible filter test for colonoscopy selection in a screening population with positive fecal immunology. Endoscopy 2014; 46: 473-478

[11] Kobaek-Larsen M, Kroijer R, Dyrvig AK et al. Back-to-back colon capsule endoscopy and optical colonoscopy in colorectal cancer screening individuals. Colorectal Dis 2018; 20: 479-485

[12] Voska M, Zavoral M, Grega T et al. Accuracy of colon capsule endoscopy for colorectal neoplasia detection in individuals referred for a screening colonoscopy. Gastroenterol Res Pract 2019; 2019: 5975438

[13] Hussey M, Holleran G, Stack R et al. Same-day colon capsule endoscopy is a viable means to assess unexplored colonic segments after incomplete colonoscopy in selected patients. United European Gastroenterol J 2018; 6: 1556-1562
[14] Baltes P, Bota M, Albert ] et al. PillCamColon2 after incomplete colonoscopy - A prospective multicenter study. World J Gastroenterol 2018; 24: 3556-3566

[15] Kroijer R, Kobaek-Larsen M, Qvist N et al. Colon capsule endoscopy for colonic surveillance. Colorectal Dis 2019; 21: 532-537

[16] Adler SN, González Lama Y, Matallana Royo V et al. Comparison of small-bowel colon capsule endoscopy system to conventional colonoscopy for the evaluation of ulcerative colitis activity. Endosc Int Open 2019; 7: E1253-E1261

[17] Ohmiya N, Hotta N, Mitsufuji S et al. Multicenter feasibility study of bowel preparation with castor oil for colon capsule endoscopy. Dig Endosc 2019; 31: 164-172

[18] Syafiq Ismail M, Semenov S, Sihag S et al. Improving quality in colon capsule endoscopy; effects of different bowel preparation regimens. Endoscopy 2020; 52: eP97

[19] Semenov S, Atiyekeogbebe R, Ismail MS et al. The addition of castor oil as a booster in colon capsule regimens significantly improves completion rates and polyp detection. Endoscopy 2020; 52: eP133

[20] Becq A, Histace A, Camus M et al. Development of a computed cleansing score to assess quality of bowel preparation in colon capsule endoscopy. Endosc Int Open 2018; 6: E844-e850 